\title{
Role of ultrastructural determinants of glomerular permeability in ultrafiltration function loss
}

\author{
Andrea Remuzzi,, Sara Conti, ${ }^{2}$ Bogdan Ene-Iordache, ${ }^{2}$ Susanna Tomasoni, ${ }^{2}$ Paola Rizzo, ${ }^{2}$ \\ Ariela Benigni, ${ }^{2}$ and Giuseppe Remuzzi ${ }^{2,3}$ \\ 'University of Bergamo, Dalmine, Italy. ${ }^{2}$ Istituto di Ricerche Farmacologiche Mario Negri IRCCS, Bergamo, Italy. ${ }^{3}$ L. Sacco \\ Department of Biomedical and Clinical Sciences, University of Milan, Milan, Italy.
}

\begin{abstract}
The epithelial filtration slit is a crucial component of the glomerular capillary membrane, which is essential for maintaining glomerular filtration function. Though chronic kidney diseases are an immense clinical problem, the mechanisms through which structural alterations reduce glomerular water filtration have not yet been understood completely. To investigate the mechanisms underlying filtration function loss, we studied rats with spontaneously occurring progressive kidney disease, either treated with angiotensin II antagonist or untreated, combining high-resolution electron microscopy of the glomerular capillary wall with theoretical water filtration modeling. Under pathological conditions, epithelial filtration pores and the extension of the subpodocyte space were larger than in normal controls. Numerical analyses indicated that these ultrastructural changes increased hydraulic resistance of the glomerular capillary wall by extending coverage of the filtration barrier by the subpodocyte space, with the changes in hydrodynamic forces acting on podocytes likely being responsible for their detachment. Angiotensin II inhibition normalized the subpodocyte space's hydraulic resistance, restored mechanical podocyte load, and preserved CD151- $\alpha 3$ integrin complex assembly, improving podocyte adherence and survival. Our results show that ultrastructural changes in podocytes are major determinants of the hydraulic resistance of the glomerular capillary wall and highlight the mechanism of podocyte loss in kidney disease progression, as well as the mechanisms underlying angiotensin II inhibition.
\end{abstract}

Authorship note: AR and SC contributed equally to this work.

Conflict of interest: The authors have declared that no conflict of interest exists

Copyright: (c) 2020, American Society for Clinical Investigation.

Submitted: February 12, 2020

Accepted: May 27, 2020

Published: July 9, 2020.

Reference information: JCI Insight. 2020;5(13):e137249.

https://doi.org/10.1172/jci.

insight.137249.

\section{Introduction}

More than 850 million people experience some form of kidney disease, which is double the number of people who have diabetes and exceeds by 20 times the number of individuals living with cancer and AIDS (1). Between 5 and 7 million people die prematurely each year because they do not have access to renal replacement therapy. To address this enormous clinical and economic problem, we need to better understand the mechanisms underlying loss of renal function, and to develop effective pharmacological treatments to halt chronic kidney disease progression.

Under normal conditions, plasma water filtration depends on the ultrastructure of the 3 layers of the glomerular filtration barrier (GFB), consisting of the endothelial cell layer (ENL), the glomerular basement membrane (GBM), and particularly the filtration slit (FS) of the podocyte foot process layer. This intercellular junction of the podocytes enables a high water filtration rate and almost complete retention of plasma proteins (2-4). In advanced renal disease, segmental and global glomerular sclerosis reduces the available filtering surface area (FSA) and water filtration, while in the initial phase in nonsclerosed glomeruli, there are changes in capillary membrane permeability to water and macromolecules that are responsible for reduced water filtration and increased protein leakage.

To better understand these pathophysiological mechanisms, we previously investigated the role of the GFB ultrastructural changes that are involved in the progressive decrease in glomerular filtration rate (GFR) in Munich Wistar Frömter (MWF) rats, an experimental model of spontaneous proteinuria and glomerulosclerosis (5-8) due to an inborn deficit of nephrons in these animals, which develop progressive renal damage resembling nonimmunological proteinuric renal diseases in human nephropathies. Using this model, we revealed the presence of ellipsoidal and circular pores in the physiological FS that are heterogeneous in size and shape (9). 
Table 1. Systemic parameters measured in Wistar, MWF and MWF-treated rats at 60 weeks of age

\begin{tabular}{|c|c|c|c|}
\hline & Wistar & MWF & MWF +lisinopril \\
\hline Body weight (g) & $477 \pm 20$ & $444 \pm 9$ & $451 \pm 3$ \\
\hline $\mathrm{SBP}(\mathrm{mmHg})$ & $128 \pm 7$ & $212 \pm 18^{B}$ & $126 \pm 10^{D}$ \\
\hline Urinary protein excretion (mg/d) & $14 \pm 3$ & $698 \pm 109^{B}$ & $258 \pm 106^{A, C}$ \\
\hline Serum creatinine (mg/dL) & $0.49 \pm 0.06$ & $1.43 \pm 0.74$ & $0.91 \pm 0.25$ \\
\hline
\end{tabular}

Values are mean $\pm \mathrm{SD} .{ }^{A} P<0.05,{ }^{\mathrm{B}} P<0.001$, vs. Wistar rats. ${ }^{\mathrm{C}} P<0.01,{ }^{\mathrm{D}} P<0.001$ vs. MWF rats.

This organization of the slits' ultrastructure was different from the zipper-like model proposed by Rodewald and Karnovsky in 1974 (10) and provided a view of the FS as a dynamic heteroporous structure. Since then, several studies have confirmed our observations $(11,12)$, demonstrating that FS structure is actually very different and far more complex than previously thought, and that it is likely a dynamic type of cell-cell contact that may influence water filtration (13).

Angiotensin II (AII)-converting enzyme inhibition (ACEi) reduced proteinuria and prevented the decrease in glomerular ultrafiltration coefficient $\left(K_{f}\right)(14)$, while it did not affect the mean size of the hypothetical glomerular membrane pores (15) but reduced the radius of a small fraction of largest pores. AII antagonism did not induce ultrastructural changes in peripheral capillary FSA, FS length and width, or GBM thickness (16). These observations did not clarify which mechanisms reduce the hydraulic permeability of the glomerular capillary wall in the MWF rat as they age or how AII antagonism ameliorates $K_{f}$ Since the progression of renal disease is similar in this model and in humans $(7,17)$, such information is crucial to understanding the pathophysiology of progressive glomerular diseases and the mechanisms of action of AII antagonists, the most widely used class of drugs in these patients (18).

In addition to improving $K_{f}$, AII antagonism prevents podocyte loss during glomerular disease progression (19). A reduction in the number of podocytes may affect their spatial organization and, particularly, the so-called subpodocyte space (SPS) within the glomerular capillary wall. The effect of AII antagonism on the volume and shape of the SPS has not been investigated in models of kidney disease, or human kidney specimens, due to the complexity of the very heterogeneous 3D spatial organization of the podocytes $(20,21)$.

The objective of our study, therefore was to investigate the glomerular determinants of water filtration that are related to loss of renal function. Combining quantitative ultrastructure investigation of the glomerular FS with numerical simulation of water flow across the different layers of the GFB, we estimated functional changes induced by glomerular disease in the whole capillary wall, and quantified the effects of AII antagonism. Finally, we also evaluated the interaction between tetraspanin (CD151) and $\alpha 3$ integrin, which are key regulatory factors of podocyte adhesion to $\operatorname{GBM}(22,23)$.

\section{Results}

Changes in systemic and kidney functional parameters and in the ultrastructure of the FS. Consistent with previous data $(9,19)$, untreated MWF rats exhibited significantly higher systolic blood pressure (SBP), proteinuria, and serum creatinine compared with Wistar rats (Table 1). Treatment with lisinopril lowered SBP to normal values, significantly reduced proteinuria compared with untreated MWF rats $(P<0.01)$, and numerically reduced serum creatinine levels.

We analyzed the ultrastructure of the FSs, estimating the size of ellipsoidal pores using previously described methods (9). Analysis of scanning electron microscopy (SEM) photomicrographs revealed an increase in mean pore dimension in proteinuric MWF compared with Wistar rats (Figure 1, F and C). As shown in Figure 1A, in Wistar rats, we estimated a mean radius for FS pores of $12.48 \mathrm{~nm}$ and mean pore area of $501 \mathrm{~nm}^{2}$, the majority of pore areas being smaller than $2.5 \times 10^{3} \mathrm{~nm}^{2}$, with larger pores (area $>2.50$ $\times 10^{3} \mathrm{~nm}^{2}$ ) representing $6.7 \%$ of the total pore area distribution and with a maximum of $4.50 \times 10^{3} \mathrm{~nm}^{2}$ (Figure 1B). In the untreated MWF group, the pore radius averaged $15.06 \mathrm{~nm}$ (Figure 1D), with a mean pore area of $780 \mathrm{~nm}^{2}$, and the percentage of larger pores was about $10 \%$ of the distribution (Figure 1E). In MWF rats treated with lisinopril, the pore size was smaller, with a mean pore radius of $11.19 \mathrm{~nm}$, a mean pore area of $420 \mathrm{~nm}^{2}$, and no pores with area larger than $2.50 \times 10^{3} \mathrm{~nm}^{2}$ (Figure 1, G-I). 


\section{A Wistar}

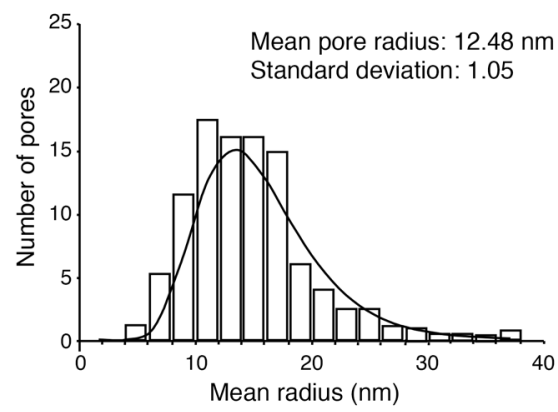

D MWF

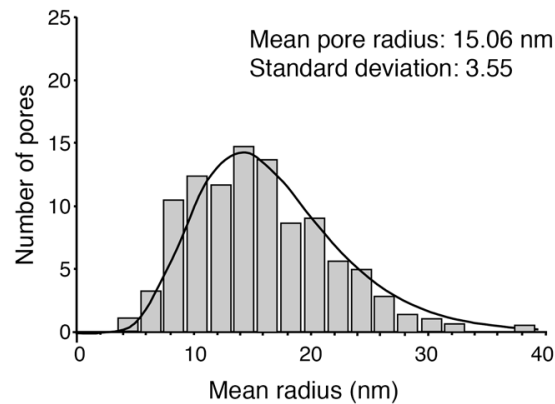

G MWF + lisinopril

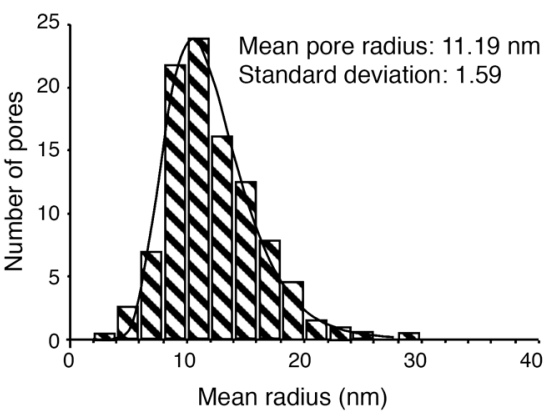

B Wistar

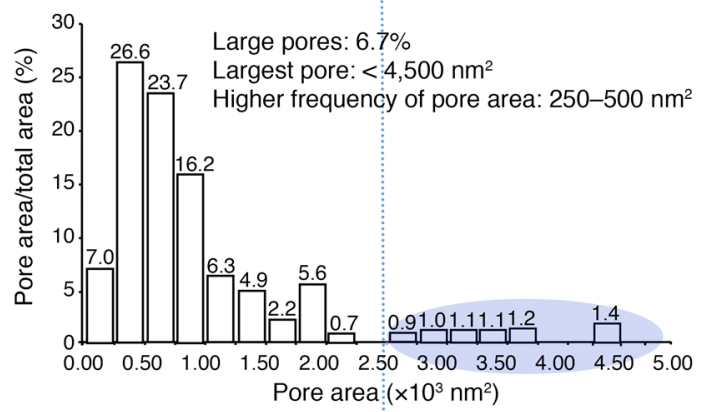

E MWF

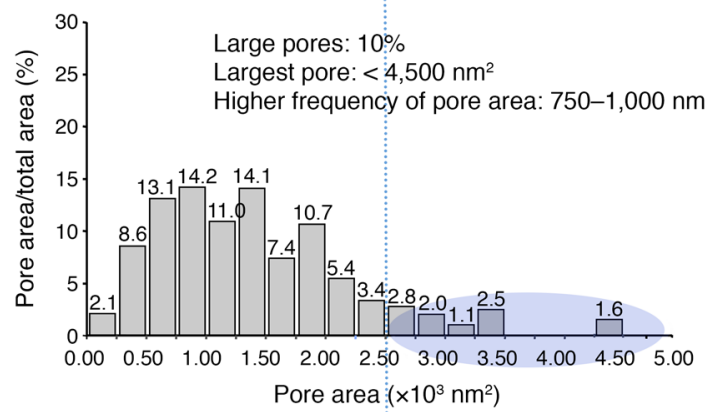

H MWF + lisinopril

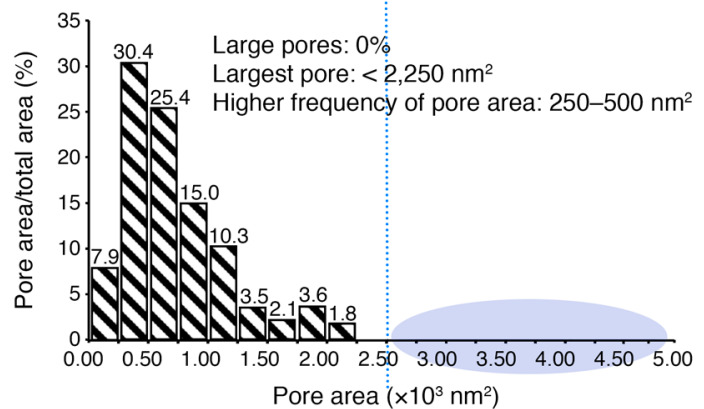

C

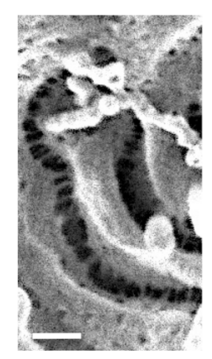

$\mathbf{F}$

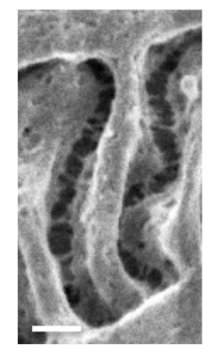

I

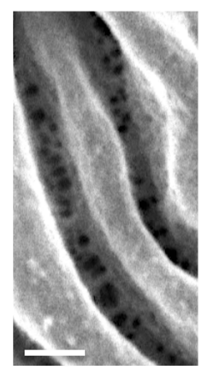

Figure 1. ACEi treatment reduced mean pore radius in MWF rats. Distribution of slit pore sizes of Wistar (A-C), MWF (D-F), and MWF rats treated with lisinopril (G-I), as measured by digital morphometrical analysis and the best fit lognormal probability distribution of pore radii. Distribution histograms of fractional pore area (\%) for Wistar (B), MWF (E), and MWF rats treated with lisinopril (H). Representative scanning electron photomicrographs of filtration pore ultrastructure in Wistar (C) and MWF rats at 60 weeks of age $(\mathbf{F})$ and MWF rats treated with lisinopril from 40-60 weeks of age (I). Scale bars: 100 $\mathrm{nm} . n=3$ rats per group were used, and 600 pores were quantified per group. The blue-shaded areas highlight the subpopulation of larger pores, with area $>2.50 \times 10^{3} \mathrm{~nm}^{2}$ (as indicated on the $x$ axis by the the blue dotted vertical lines).

As shown in Table 2, pore frequency in untreated MWF rats was lower than in Wistar rats, while GBM thickness and distance between pores were significantly greater in MWF than in Wistar rats. After treatment with lisinopril, the pore frequency and distance between pores in MWF rats were comparable to those in control rats, and GBM thickness remained similar to that of untreated MWF rats. No differences were observed between groups regarding the shape of pores $(R / r)$. We also estimated the extent of SPS by measuring its area density (assumed to be equal to the volume density) (16) using transmission electron microscopy (TEM) images of glomeruli from Wistar and MWF rats at 20 weeks of age, in which structural alterations in podocytes have already developed, but in the absence of glomerulosclerosis (8). As shown in Figure 2, A and B, the volume density of SPS was significantly higher in MWF than in Wistar rats (averaging 3.37\% and $1.51 \%$, respectively, in Wistar and MWF rats, $P<0.01$ ). Treatment with lisinopril reduced the SPS volume (2.16\%).

Since the increase in SPS in MWF rats may have increased the mechanical forces exerted on these cells by water filtration and induced podocyte detachment from the GBM, we identified detached podocytes 
Table 2. Morphometric characterization of epithelial slit pores and GBM in Wistar, MWF, and treated MWF rats

\begin{tabular}{|c|c|c|c|c|}
\hline Group & Frequency of pores ( $n$ per $\mu \mathrm{m}$ of GBM) & Distance between pores $(\mathrm{nm})$ & $R / r$ & GBM thickness ( $\mu \mathrm{m})$ \\
\hline Wistar & 12.9 & $27.02(4.7-200)$ & 1.65 & $0.217 \pm 0.12$ \\
\hline MWF & 8.75 & $47.99(6.3-249)^{A}$ & 1.36 & $0.286 \pm 0.13^{\mathrm{B}}$ \\
\hline MWF+lis & 12.87 & $27.58(6.3-162)^{c}$ & 1.18 & $0.283 \pm 0.07^{A}$ \\
\hline
\end{tabular}

Values are median (range) or mean \pm SD. The distance between pores was expressed either as the mean or as a range of distances between pores (in parentheses). $R / r$ is the ratio between major $(R)$ and minor $(r)$ pore radii. ${ }^{A} P<0.01,{ }^{B} P<0.001$ vs. Wistar rats; ${ }^{C} P<0.01$ vs. MWF rats.

floating free in the Bowman's space and in the lumen of proximal tubules (Figure 2, C and E), as confirmed by toluidine blue-stained sections (Figure 2, D and F). Detached podocytes found in MWF rats were apparently viable, with intact nuclei and without signs of apoptosis $(24,25)$.

Hydraulic resistance to water filtration of the GFB. We estimated the hydraulic resistance of the whole glomerular capillary wall ( $\mathrm{R}_{\text {тот }}$ ), using micropuncture data and glomerular FSA as previously reported $(14,26)$, for the 3 groups of rats (see Supplemental Material; supplemental material available online with this article;

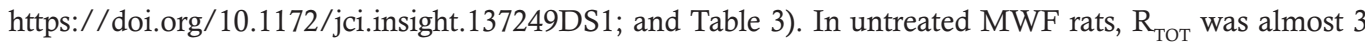
times higher than in the control Wistar group, while treatment of MWF rats with lisinopril restored it to a normal value. Using ultrastructural parameters to build 3D numerical models (see Figure 3 and Table 4) and computational fluid dynamics (CFD) analysis, we also estimated the resistance to water flow of the GFB, which is the reciprocal of the hydraulic permeability reported in Supplemental Table 2. The CFD estimated that the hydraulic resistance of GFB alone (without contribution of the SPS) is lower than that of the total glomerular capillary wall, especially for MWF rats (Table 3). We then estimated the additional contribution to the total resistance of the glomerular capillary wall by the presence of the SPS, using the model of Neal

A

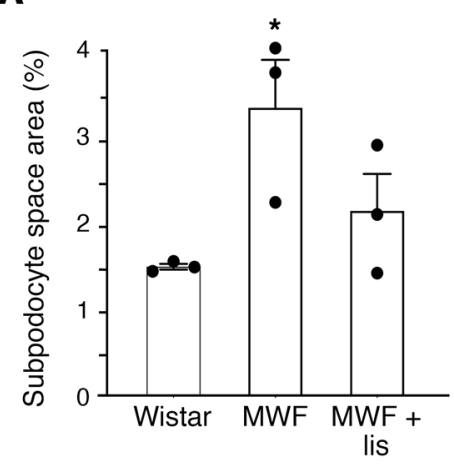

B

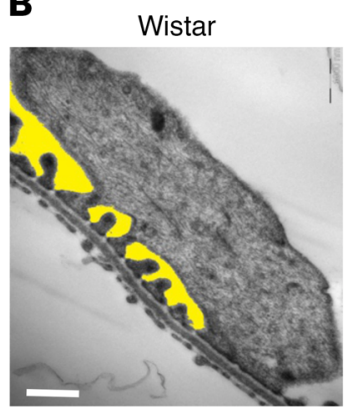

MWF

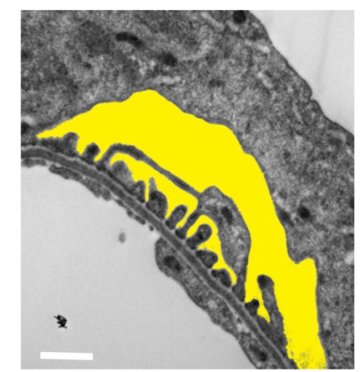

MWF + lis

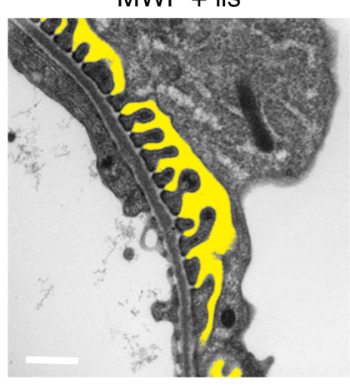

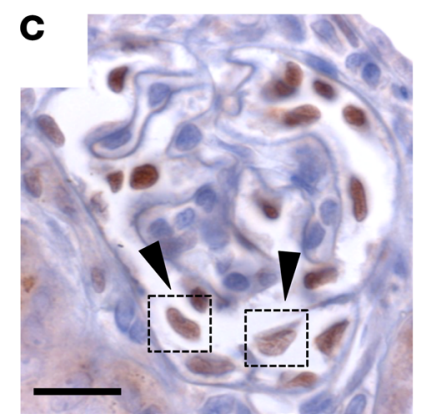
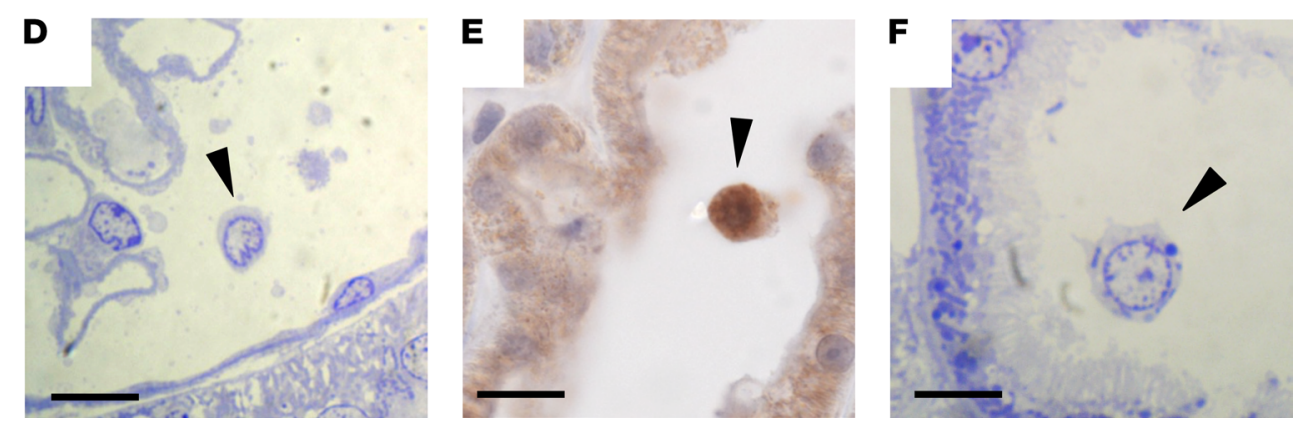

Figure 2. ACEi reduced SPS in proteinuric MWF animals. (A) Quantification of SPS area. ${ }^{*} P<0.05$ vs. Wistar; ANOVA with Tukey's post hoc test. lis, lisinopril. (B) Representative images from Wistar and MWF rats at 20 weeks and in MWF rats treated with lisinopril between 10 and 20 weeks of age. The SPS is highlighted in yellow. Scale bars: $500 \mathrm{~nm}$. Images are representative of 3 rats per group. In proteinuric MWF animals, podocytes are lost by detaching from the GBM as viable cells. Immunoperoxidase staining for WT-1 ( $\mathbf{C}$ and $\mathbf{E}$ ) and toluidine blue-stained sections in MWF rats ( $\mathbf{D}$ and $\mathbf{F}$ ) showing detached podocytes with viable-appearing nuclei (arrowheads) in the Bowman's space (C and $\mathbf{D}$ ) and in the tubular lumen (E and $\mathbf{F}$ ). Images are representative of 3 rats per group, and 10-12 glomeruli were analyzed per rat. Scale bars: $20 \mu \mathrm{m}$ for $\mathbf{C}, \mathbf{E}$, and $\mathbf{F} ; 10 \mu \mathrm{m}$ for $\mathbf{D}$. 
Table 3. Measured total hydraulic resistance $\left(R_{T O T}\right)$ of the glomerular capillary wall, and calculated resistance of the $G F B\left(R_{\mathrm{GFB}}\right)$, fractional area covered by SPS $\left(\varepsilon_{c}\right)$, pressure in the SPS, and hydrodynamic force acting on a single podocyte

\begin{tabular}{|c|c|c|c|c|c|c|c|}
\hline & $R_{\text {TOT }}\left(\times 10^{8} \mathrm{Pz}\right.$ & <10 ${ }^{8} \mathrm{~Pa} \mathrm{~s} \mathrm{m^{-1 } )}$ & $\varepsilon_{c}$ & $\mathbf{P}_{\mathrm{Gc}}(\mathrm{mmHg})$ & $\mathbf{P}_{\mathrm{sPS}}(\mathrm{mmHg})$ & $\mathbf{P}_{\mathrm{BS}}(\mathrm{mmHg})$ & $F_{\mathrm{POD}}(\mu \mathrm{N})$ \\
\hline Wistar (ref. 26) & 2.73 & 2.33 & $\begin{array}{c}0.192 \\
{[0.158-0.253]}\end{array}$ & 44 & 37 [42-31] & 12 & $0.4-1.0$ \\
\hline MWF (ref. 14) & 7.43 & 2.84 & $\begin{array}{c}0.689 \\
{[0.632-0.794]}\end{array}$ & 51 & $47[50-43]$ & 13 & $14.8-22.9$ \\
\hline MWF + lis (ref. 14) & 3.28 & 2.66 & $\begin{array}{c}0.253 \\
{[0.203-0.345]}\end{array}$ & 44 & 36 [42-30] & 13 & $1.0-2.8$ \\
\hline
\end{tabular}

$\mathrm{Pa}$, pascal; $\mathrm{F}_{\mathrm{POD}}$, force acting on a single podocyte; $\mathrm{P}_{\mathrm{CC}}$, pressure in the glomerular capillary.

and coworkers, described in detail in the supplemental material. This model assumes overall resistance as the parallel of 2 resistances, that of the naked GFB and that of the GFB covered by SPS, as a function of the parameter $\varepsilon$, the ratio between the surface area of GFB covered by SPS and the total FSA. Since specific input parameters of the model of SPS resistance, i.e., length $(\delta)$ and diameter $(b)$ of SPS exit pores (SEPS), are not directly measurable, we used sensitivity analysis to derive a range of values of $\varepsilon$ that allow obtaining total resistance of the capillary wall equal to that derived from micropuncture data (Figure 4$)$. The parameter $\varepsilon$ estimated by the sensitivity analysis averaged $0.19,0.69$, and 0.25 for the 3 groups, respectively (see ranges of $\varepsilon$ in Table 3). Thus, in MWF rats, SPS enlarged to cover almost $70 \%$ of the FSA, while SPS covered less than $20 \%$ of FSA in Wistar rats. The effect of lisinopril treatment was to reduce this covered FSA to values near those estimated for the normal control group. In addition, we calculated that in normal rats, the relative contribution of the FSA covered by SPS to the total $K_{f}$ is on average $5 \%$, with a contribution of the naked GFB of $95 \%$. In MWF rats the contribution of the FSA covered by SPS increased up to $18 \%$, with a relative contribution of the uncovered part of FSA of $82 \%$. In contrast, in treated rats the relative contribution of the SPS-covered portion to overall ultrafiltration was reduced to $8 \%$, with the contribution of the naked GFB averaging $92 \%$, indicating that AII inhibition decreases SPS spatial extension, as well as its contribution to the hydraulic resistance of the glomerular capillary wall.

The values of estimated $\varepsilon$ are consistent with the ultrastructure of the glomerular filtration capillary wall in younger rats (Figure 2B). In the MWF rat, SPS is expanded in volume and covers large areas of the GBM, forming wide spaces between the podocyte's body and the underlying filtering membrane. These important changes in the 3D structure of the SPS are likely responsible for the increased coverage of the FSA by the SPS $(\varepsilon)$ we estimated for rats in the pathological condition. In contrast, in normal Wistar and in treated MWF rats, the spatial extension of the SPS was much lower (Figure 2B).

Using the theoretical model of Neal et al., it was possible also to calculate hydraulic pressure within the SPS $\left(\mathrm{P}_{\mathrm{SPS}}\right)$. According to the different assumed input parameters, the values of $\mathrm{P}_{\mathrm{SPS}}$ are in the range of 31-42 mmHg for normal Wistar rats, 43-50 mmHg for MWF rats, and between 30 and $42 \mathrm{mmHg}$ in treated MWF rats. On the basis of these values, and those of $\varepsilon$ estimated for the 3 groups, we also calculated the average physical force acting on each podocyte that tends to detach these cells from the capillary membrane. The absolute force to which the podocyte is exposed is the result of the pressure applied underneath and above the cells, which are the $\mathrm{P}_{\mathrm{SPS}}$ and hydraulic pressure in Bowman's space $\left(\mathrm{P}_{\mathrm{BS}}\right)$. The force can be calculated as the product of the pressure difference multiplied by the average SPS surface area of a single podocyte. We estimated this surface area by dividing total $\mathrm{A}_{\mathrm{SPS}}$ (equal to FSA $\times \varepsilon$ ) by the mean number of podocytes per glomerular tuft that was previously reported for Wistar and MWF rats, as well as for MWF rats treated with lisinopril (19). The mean number of podocytes that we assumed for the 3 groups was 159, 58, and 144 podocytes/glomerulus, respectively. As reported in Table 3, the calculated average force acting on podocytes in normal rats, for the estimated range of $\varepsilon$ and pressure values, was in the range of 0.4-1.0 $\mu \mathrm{N}$. The calculated force for the MWF rats was much higher than normal values and in the range of $14.8-22.9 \mu \mathrm{N}$. Of interest, lisinopril treatment almost normalized the average physical force acting on a single podocyte to $1.0-2.8 \mu \mathrm{N}$.

Podocyte CD151- $\alpha 3$ integrin interaction in MWF rats and the effect of $A C E$ inhibition. To further examine the molecular mechanism underlying the detachment and spatial organization of podocytes, we evaluated the expression of $\alpha 3$ integrin and CD151 $(22,27)$ - a tetraspanin protein that interacts directly with $\alpha 3$ integrin at 
A
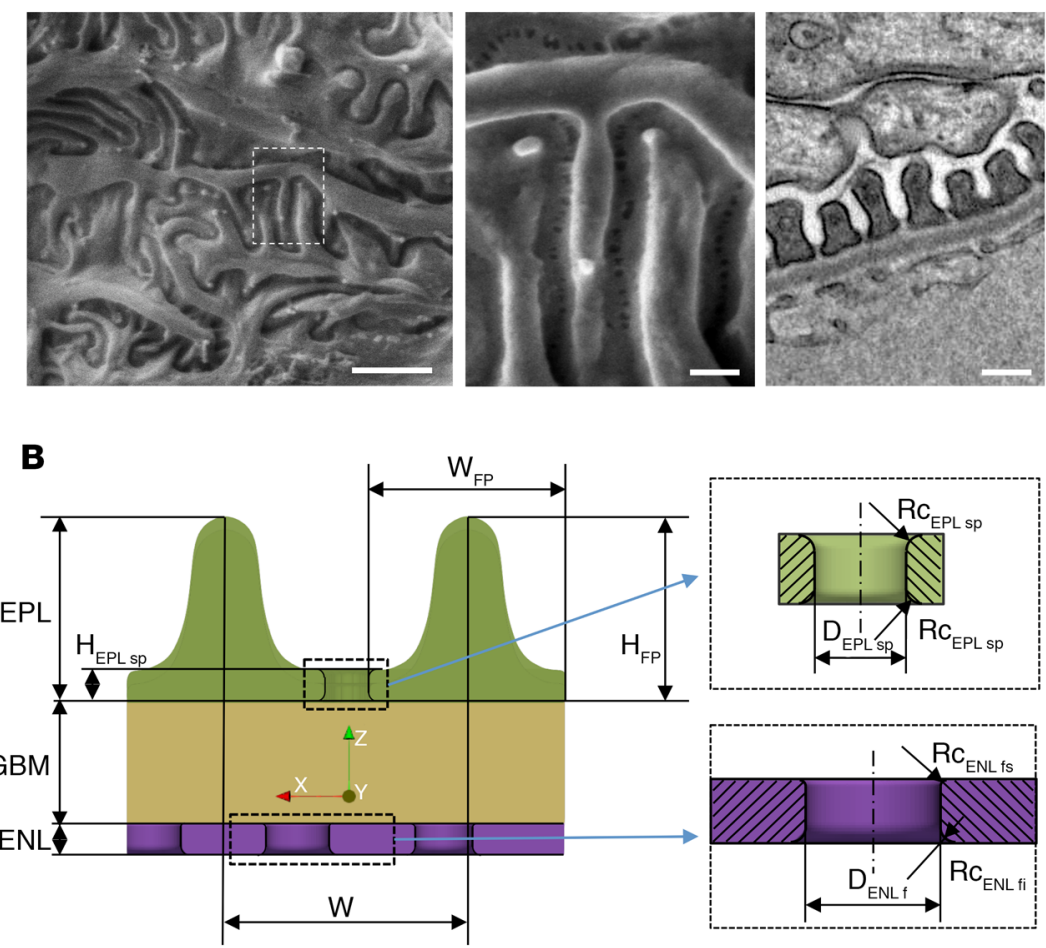

C
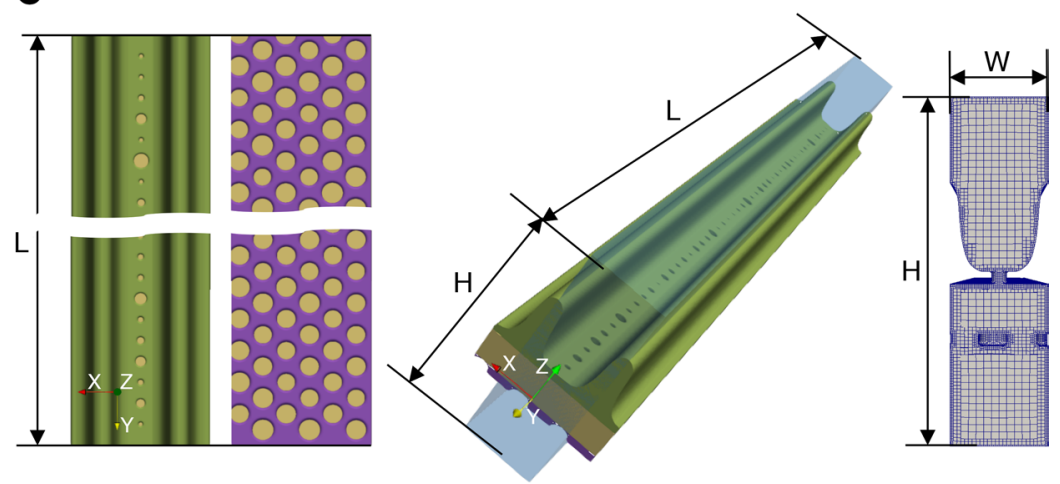

Figure 3. 3D model of the unit cell of GFB based on a single FS. (A) Representative scanning electron photomicrographs of FS ultrastructure at low (left, scale bar: $1 \mu \mathrm{m}$ ) and high magnification (middle, scale bar: $200 \mathrm{~nm}$ ); high-resolution transmission electron photomicrograph of glomerular capillary membrane (right, scale bar: 250 $\mathrm{nm}) . n=3$ rats per group. (B) The model consists of 3 layers: ENL, GBM, and EPL; the cell is periodic in the $x$ and $y$ directions, while filtration occurs in the positive $z$ direction (left). Insets show details of ENL and EPL pores (right). (C) Top and bottom views showing EPL pore and ENL fenestra size and distribution (left). 3D view of the computational model: the blue box represents the fluid domain of width $(W) \times$ length $(L) \times$ height $(H)$ dimensions (middle). Section view of the fluid mesh used for CFD simulation (right). For the definitions and values of geometrical parameters, see Table 4.

the base of podocyte foot processes, mediating adhesion to the GBM (28-30). Compared with normal Wistar rats, MWF rats exhibited significantly less glomerular CD151 and $\alpha 3$ integrin expression $(P<0.01$, Figure 5). In order to determine whether the interaction between CD151 and $\alpha 3$ integrin is compromised in MWF rats, we used an immunofluorescence double-labeling approach. In proteinuric MWF rats, the colocalization of CD151 and $\alpha 3$ integrin was remarkably lower. In contrast, in MWF rats treated with lisinopril, CD151 and $\alpha 3$ integrin expression was partially restored (Figure 5A). We then used the Pearson's correlation coefficient and Manders's overlap coefficient, to quantify the colocalization between the 2 fluorophores. Both analyses clearly showed that colocalization of CD151 with $\alpha 3$ integrin was significantly lower in glomeruli of untreated MWF compared with normal Wistar rats, while it was normalized in MWF rats treated with lisinopril (Figure 5B). 
Table 4. Ultrastructural glomerular parameters assumed for the model

\begin{tabular}{|c|c|c|c|c|c|c|c|c|c|c|c|c|c|}
\hline & $\underset{\text { (ref. 40) }}{\mathrm{T}_{\mathrm{ENL}}}$ & $\begin{array}{c}D_{\text {ENLf }} \\
\text { (ref. 12) }\end{array}$ & $\mathbf{R c}_{\mathrm{ENLfi}}$ & $\mathbf{R C}_{\text {ENL fs }}$ & $T_{\text {GBM }}{ }^{A}$ & $\begin{array}{c}\mathbf{H}_{\text {EPL sp }} \\
\text { (ref. 40) }\end{array}$ & $D_{\text {EPL sp }}{ }^{A}$ & $\underset{\text { (ref. 16) }}{\mathbf{H}_{\mathrm{Fp}}}$ & $\begin{array}{c}W_{F P} \\
\text { (ref. 16) }\end{array}$ & $\mathbf{R c}_{\text {EPL sp }}$ & $\mathbf{w}$ & $\mathbf{L}$ & H \\
\hline Wistar & 50 & $\begin{array}{c}100 \\
(90-110)\end{array}$ & 16 & 5 & 217 & 50 & $25(12-76)$ & 250 & 200 & 10 & 310 & 3875 & 1417 \\
\hline $\mathrm{MWF}+$ lis & 50 & $\begin{array}{c}100 \\
(90-110)\end{array}$ & 16 & 5 & 283 & 50 & $22(12-52)$ & 288 & 210 & 10 & 370 & 3885 & 1483 \\
\hline
\end{tabular}

All values are in $\mathrm{nm}$. A Data acquired in this study. $\mathrm{T}_{\mathrm{ENL}}$, endothelial layer thickness; $\mathrm{D}_{\mathrm{ENL} \text { f }}$, diameter of endothelial fenestrae; $\mathrm{RC}_{\mathrm{ENL} \text { fs }}$, radius of curvature of endothelial fenestrae toward the GBM; $\mathrm{Rc}_{\mathrm{ENLFi}}$, radius of curvature of endothelial fenestrae toward the capillary lumen; $\mathrm{T}_{\mathrm{CBM}}$, thickness of $\mathrm{GBM}$; $\mathrm{H}_{\mathrm{EPL} \text {. } p}$, height of epithelial slit pores; $D_{E P L ~ s p}$, diameter (and range) of epithelial slit pores; $H_{F P}$, height of the foot process; $W_{F P}$, width of the foot process; $R c_{E P L ~ s p}$, radius of curvature of epithelial slit pores.

\section{Discussion}

In the present study, we combined electron microscopy imaging and theoretical modeling of water filtration to shed light on the mechanisms underlying renal function loss in progressive kidney diseases and the effect of ACEi in preventing disease progression. Our results suggest that early alterations of podocyte morphology lead to changes in local hydraulic resistance and pressure within the glomerular capillary wall, determining the progressive loss of glomerular filtration function. ACEi, on the other hand, acts on podocyte structure, reducing the spatial extension of SPS and restoring hydraulic resistance, thus achieving near normalization of water filtration and of glomerular barrier function.

In untreated MWF rats at 60 weeks of age, when the ultrastructural alterations are expected to be developed, our observation of a subpopulation of very large FS pores confirmed previous estimations of the size of hypothetical membrane pores (14) and may explain the abnormal filtration of plasma proteins under disease conditions, thus reconciling functional data with ultrastructural evidence. Moreover, the absence of very large pores in lisinopril-treated MWF rats demonstrates that ACEi reduces proteinuria by restoring the FS pores' normal size. Previous and present data indicate that drugs that antagonize AII induce ultrastructural changes in glomerular FS pores that affect both their dimension and density, and this effect is likely responsible for the reduction in the filtration of plasma proteins such as albumin.

Using CFD analysis and morphometrical evaluation of the ultrastructure, we simulated water flow across numerical models of the GFB in control Wistar rats and estimated a hydraulic resistance that accounts for $85 \%$ of total capillary wall resistance, calculated using micropuncture data. The remaining $15 \%$ of the total resistance of the capillary wall was attributable to the presence of SPS of a portion of the FSA. In diseased animals (untreated MWF rats), the GFB resistance estimated by CFD accounted for only $38 \%$ of the total capillary wall resistance, suggesting a more important role for the SPS in restricting water filtration. We then used a theoretical approach to compute hydraulic resistance of the capillary membrane in the presence of the SPS, assuming different values of the ratio between SPS area and the FSA $(\varepsilon)$. Due to uncertainty regarding the dimensions of the length $(\delta)$ and diameter $(b)$ of the SPS, we used different values of these parameters in the model. The results of the computations showed that estimated values of $\varepsilon$ are higher in MWF rats as compared with normal rats. This result is in line with the estimation of the SPS volume density we experimentally determined in these rat strains (see Figure 2). When animals were treated with the ACE inhibitor lisinopril, FSA and $K_{f}$ remained higher than in Wistar rats (as reported in Supplemental Table 1). This is likely because MWF rats have a reduced number of nephrons that must remain enlarged to provide enough FSA for water filtration. As reported previously, AII inhibition also increased $K_{f}(14)$, once again to allow water filtration with reduced nephron numbers. Analysis of data from ACE inhibitor-treated rats showed that extension of SPS over FSA $(\varepsilon)$ is lower than in untreated MWF rats and almost comparable to that in normal Wistar rats, in line with the estimation of the SPS volume reported in Figure 2.

Increased coverage of the FSA by SPS (represented as $\mathrm{A}_{\mathrm{SPS}}$ ) is expected to increase the physical forces acting on podocytes due to shear stress and hydraulic pressure that develop in the SPS, which may increase hydraulic resistance and podocyte detachment, as previously suggested (31). Indeed, on the basis of the computed pressure in the SPS and the area of the SPS, we estimated that each cell is on average subjected 

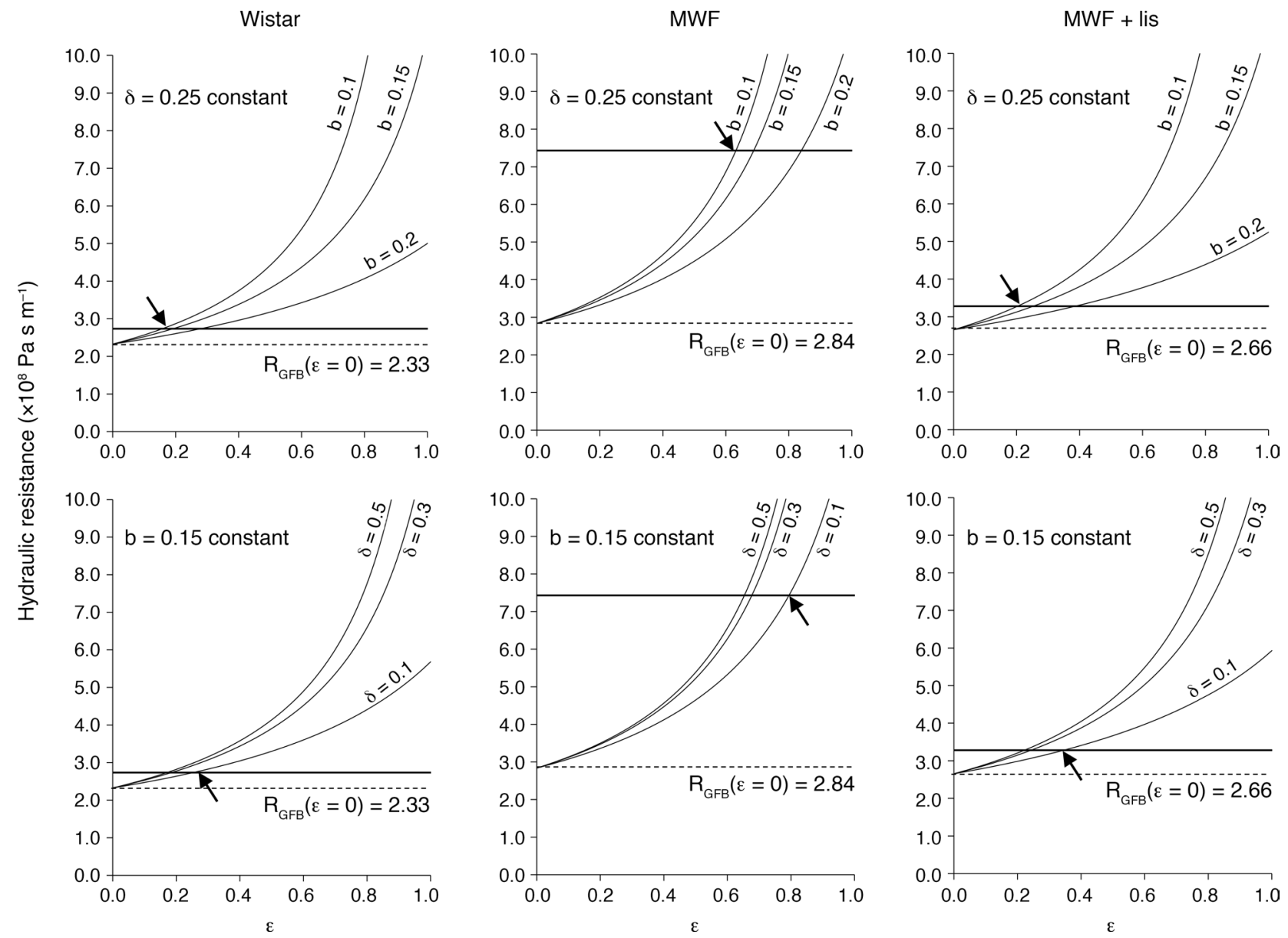

Figure 4. The higher hydraulic resistance of the glomerular capillary wall in MWF rats is due to a greatly increased resistance of the SPS. Total hydraulic resistance as function of SEP diameter $b$ (upper panels) and as function of SEP length $\delta$ (lower panels). Arrows indicate the extremities of the $\varepsilon$ range presented in Table 4 .

to a physical force more than 20 times that acting on a podocyte of a normal Wistar rat. While normal podocytes are subjected to a force around $1.0 \mu \mathrm{N}$, the podocytes of MWF rats are subjected to a detaching force in the range of $14-22 \mu \mathrm{N}$. It is very likely that the reduced number of podocytes, we and others have previously reported in glomerular diseases (19), is the result of podocyte detachment caused by hydraulic forces. In line with this hypothesis, we directly observed detachment of viable podocytes in untreated MWF rats, as shown in Figure 2. This hypothesis has been also raised previously on the basis of morphological observations (31), and the present investigation allowed us to quantitatively support the hypothesis and demonstrate that (as shown in Figure 6) podocyte detachment must be related to hydrodynamic forces emerging from reduced podocyte number, SPS volume expansion, and increased hydraulic resistance and hydraulic pressure underneath the podocyte. Our data also provide an explanation for previous studies $(20,32)$ that suggested this sequence of events, relating the ultrastructure of SPS to glomerular membrane hydraulic permeability. Here we confirmed that there is a direct effect of the hydrodynamic forces that lead to podocyte loss.

Our results show that ACE inhibition importantly reduced the resistance of the SPS and the estimated detaching force acting on single podocytes. In this condition, the filtration function was preserved, and this was associated with lower protein excretion, also suggesting a beneficial effect on protein retention by the capillary wall. These beneficial effects of ACE inhibition are likely the result of the higher number of podocytes that are present in treated animals due to reduced podocyte loss.

The mechanical resistance of podocytes to hydrodynamic load depends on proteins, the integrins that are responsible for cell anchorage to the GBM and play a key role in maintaining podocyte structure $(33,34)$. A large number of experimental and clinical studies have demonstrated that mutations of 
A
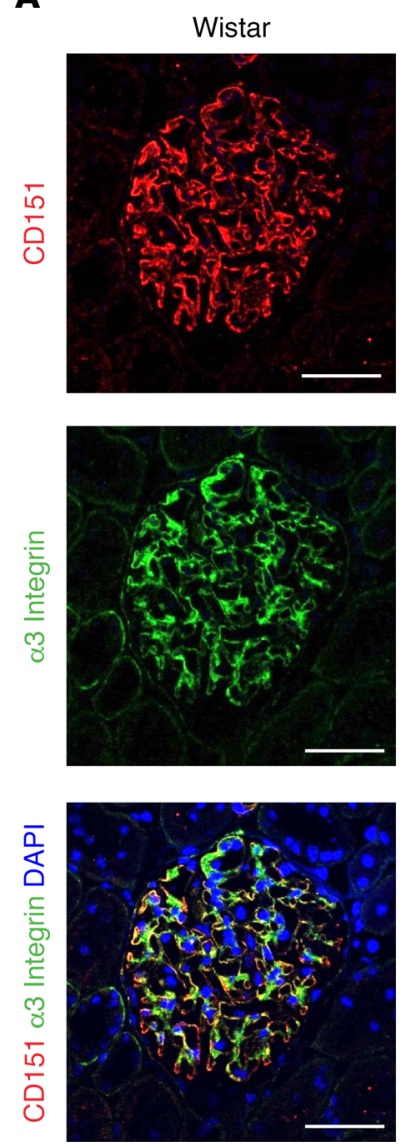

MWF
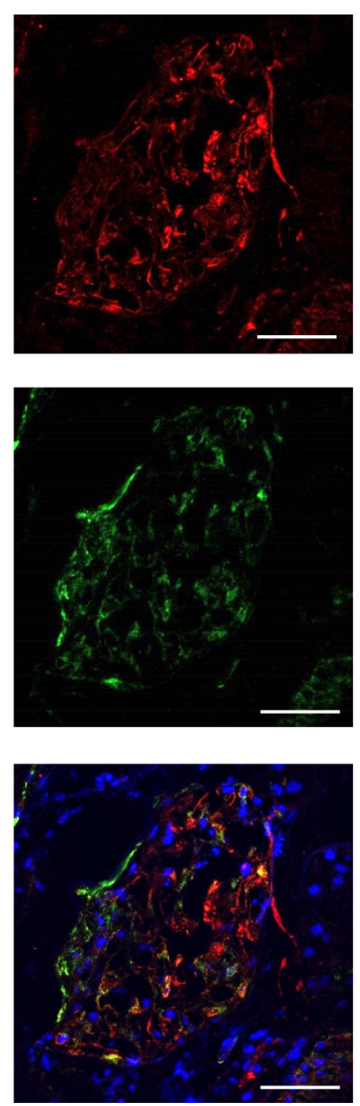

MWF + lisinopril
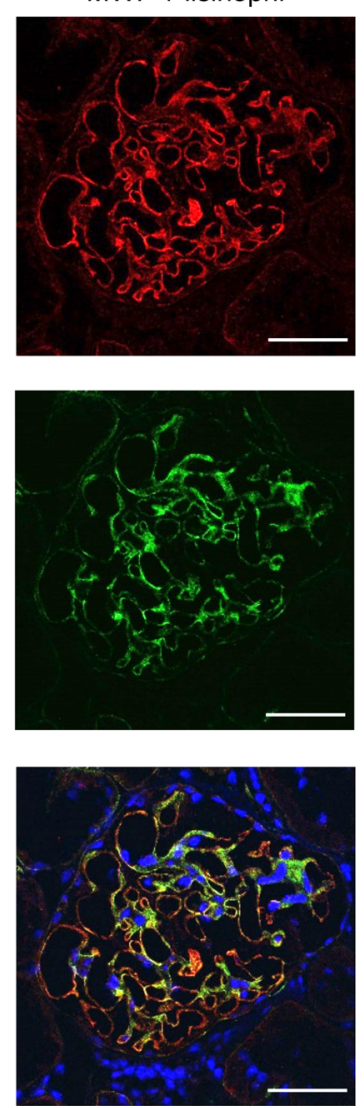

B
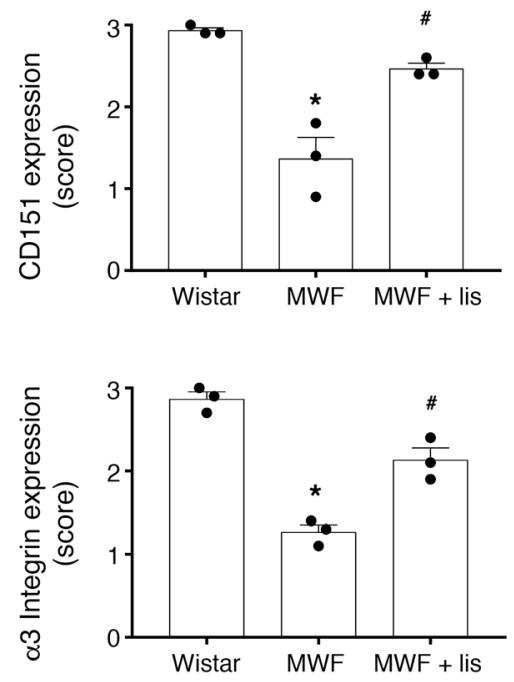

$\square$ Pearson Manders

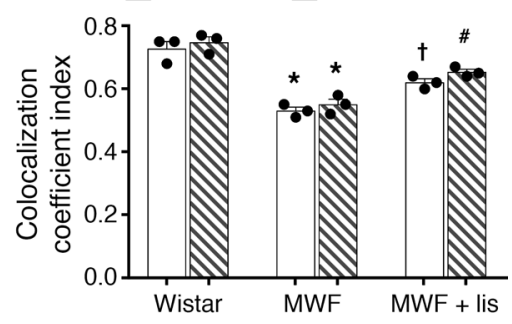

Figure 5. MWF rats exhibited reductions in CD151, $\alpha 3$ integrin, and their colocalization; and lisinopril restored CD151- $\alpha 3$ integrin interaction in proteinuric MWF animals. (A) Representative images of immunofluorescence staining for CD151 (red) and $\alpha 3$ integrin (green) and their colocalization in Wistar, MWF, and MWF rats treated with lisinopril. DAPI (blue) stains nuclei. Scale bars: $50 \mu \mathrm{m}$. (B) Expression of CD151 and $\alpha 3$ integrin (score) and their colocalization in Wistar, MWF, and MWF rats treated with lisinopril. Quantification of CD151- $\alpha 3$ integrin colocalization is represented as Pearson's and Manders's coefficients. ${ }^{*} P<0.01$ vs. Wistar, ${ }^{\dagger} P<0.05$ vs. MWF, ${ }^{\#} P<0.01$ vs. MWF; ANOVA with Tukey's post hoc test. For all staining, $n=3$ rats per group, and 10 glomeruli were quantified per rats.

integrins ( $\alpha 3$ and $\beta 1$ subunits) provoke podocyte abnormalities, with subsequent apoptosis and progressive podocyte loss, leading to focal glomerulosclerosis (FSGS) $(35,36)$. CD151 is a tetraspanin protein that interacts directly with $\alpha 3$ integrin at the base of podocyte foot processes and is a crucial modifier of integrin-mediated adhesion of podocytes to the GBM (22). Mice deficient in CD151 develop kidney abnormalities with massive proteinuria, disorganization of the GBM, and FSGS (30). Deletion of CD151 in cultured murine podocytes results in a marked reduction in podocyte adhesion (27). In humans, CD151 mutations cause nephrotic syndrome, leading to end-stage renal failure (37). Furthermore, recent evidence has demonstrated that CD151 helps podocytes stick in high-pressure conditions, increasing the tightness of integrin $\alpha 3 \beta 1$-dependent adhesion to the GBM (38). Considering the hemodynamic changes that characterize MWF rats, we explored the interaction between CD151 and $\alpha 3$ integrin and demonstrated that the physical interaction between CD151 and $\alpha 3$ integrin was defective in untreated MWF rats, while ACEi protected the CD151- $\alpha 3$ integrin tethering interaction (Figure 6). Our observations are in line with experimental evidence showing that drugs able to lower systemic and intraglomerular blood pressure, such as ACE inhibitors, are protective in a murine model of CD151 deficiency, whereas treatment with drugs only able to decrease systemic pressure did not show any protection from glomerular disease. In the treated MWF rats, ACEi may have increased the resistance of podocytes in their adhesion to the GBM. In addition, our data strongly indicate that it is very important to start treatment with AII antagonists of progressive kidney diseases as early as possible, because podocytes do not proliferate, and once certain a number of them is lost, irreversible glomerular damage takes place, leading to complete loss of renal function. 
A MWF
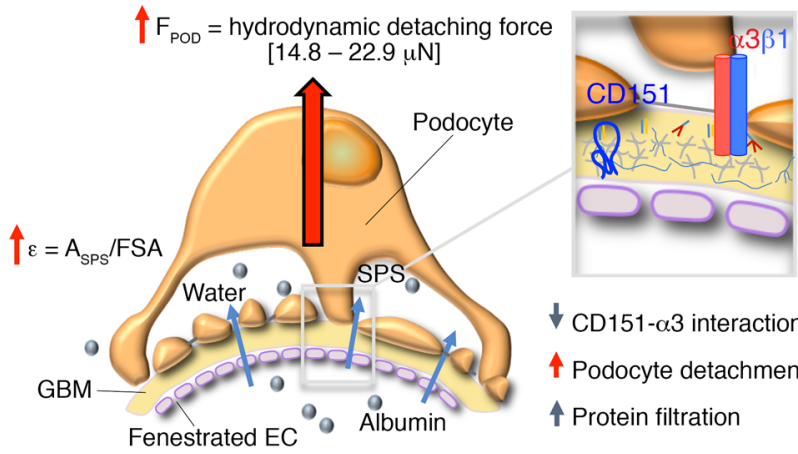

$\downarrow$ CD151- $\alpha 3$ interaction

4 Podocyte detachment

$\uparrow$ Protein filtration

\section{B MWF + ACEi}
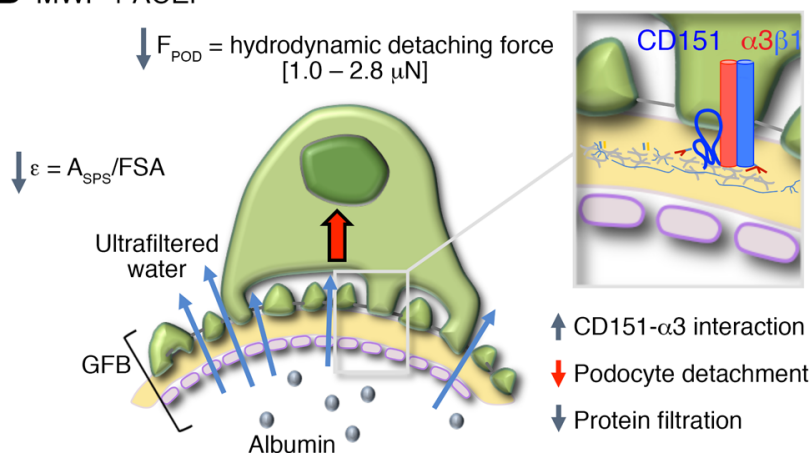

C

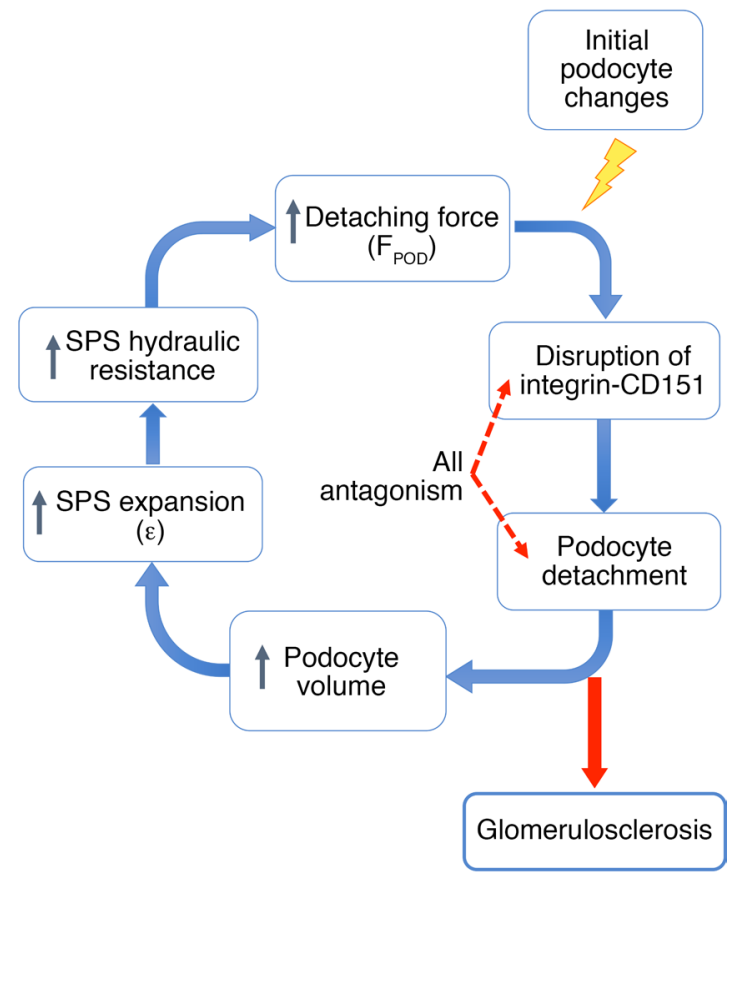

Figure 6. Schematic representation of the cascade of events that lead to changes in glomerular capillary wall function as a consequence of podocyte changes. (A) In untreated MWF rats, the hemodynamic stress induced by expanded SPS exposes podocytes to an increase in the hydrodynamic detaching forces that tend to detach podocytes from the GBM, disrupting CD151- $\alpha 3$ integrin complex assembly, with changes that reduce water filtration and facilitate albumin filtration. (B) In ACE inhibitor-treated MWF rats, CD151- $\alpha 3$ integrin complex assembly is preserved and SPS expansion is reduced, so the physical forces acting on podocytes through filtered water are lowered and podocyte loss is limited, preserving the permselective properties of the glomerular capillary wall to albumin filtration. (c) Schematic diagram of the pathogenesis of progressive glomerular disease toward glomerulosclerosis. Initial podocyte changes activate a vicious cycle in the glomerular capillary membrane, wherein disruption of CD151- $\alpha 3$ integrin complex assembly leads to podocyte detachment and the expansion of SPS, which in turn causes an increase in SPS hydraulic resistance and in the resulting detaching force, thereby perpetuating podocyte depletion and glomerulosclerosis.

Even though the boundary condition values for the CFD simulations, based on $K_{f}$ and the filtration rate of single nephron, were not measured directly here, they were carefully estimated based on previous micropuncture and kidney functional data obtained in the same rat strains. Our 3D models of the unit cell of the GFB considered different group-specific values of GBM thickness and values of Darcy permeability that were previously determined (39) in the same animal groups. Given the absence of experimental data in the literature, we assumed a similar water permeability value $\left(K_{D}=2.7 \mathrm{~nm}^{2}\right)(40)$ for the glycocalyx layer in the endothelial fenestrae in all 3 groups. It can be argued that properties of the glycocalyx layer can differ in different rat strains with age and treatment; however, we estimated that the contribution of endothelial layer to GFB permeability was only around $15 \%$ (see Supplemental Table 2). Thus, these potential changes may have little effect of the permeability properties of the entire capillary wall. Additionally, the analytical model to estimate the contribution of the SPS to total hydraulic resistance of the glomerular capillary wall is based on inherent assumptions that need to be mentioned as well. Our model, in line with that of Neal et al. (32), assumes that the height of the SPS is uniform along its whole length; that fluid could exit only at a constant radius (a); and that SEPs are cylindrical, such that flow follows Poiseuille's principle of resistance (32). Also, in order to estimate the average hydrodynamic force acting on a single podocyte, we assumed a mean podocyte number per glomerulus tuft not counted in the present study, but taken from our previous investigation rats of the same strain, matched for age (19). Additional investigations on these structural and functional parameters may overcome these limitations and make theoretical calculation even more reliable. However, our study made it possible, for the first time to our knowledge, to find an alternative and successful way to effectively estimate the functional role of SPS in the GFB, considering it as a stand-alone layer that adds resistance to filtration of water while passing from the GBM to the Bowman's space. 
In conclusion, our study provides evidence that loss of glomerular filtration function in experimental glomerular injury strongly depends on the disarrangement of podocytes, which causes an expansion of the SPS, an increase in its hydraulic resistance to filtered water, and increased force acting within the SPS that tends to detach podocytes from the capillary membrane. ACEi preserves podocyte structure, reducing the spatial extension of SPS and restoring hydrodynamic detaching forces on podocytes, ultimately normalizing water filtration and glomerular barrier function. These effects were associated with the preservation of the $\mathrm{CD} 151 / \alpha 3$ integrin axis, with a consequent stronger link between podocytes and the GBM. Our results encourage the use of ultrastructural analysis and theoretical modeling to investigate the pathophysiology of glomerular diseases and the effects of drugs to prevent renal function loss. The knowledge that glomerular ultrafiltration loss is related to mechanical failure of podocytes can lead to more effective strategies to prevent podocyte loss and renal disease progression, and ultimately to address this global clinical and social problem.

\section{Methods}

Animals. This study was performed on kidney samples from male MWF rats from our colony (8) and from male Wistar rats (Charles River) used as normal controls ( $n=3$ /group). Wistar and MWF rats were studied at 60 weeks of age. An additional group of MWF rats $(n=3)$ was treated with lisinopril $(12.5 \mathrm{mg} / \mathrm{L}$ in the drinking water) between 40 and 60 weeks of age. Animals were maintained in a temperature-controlled room regulated with a 12-hour light/12-hour dark cycle, and had free access to water and food. At sacrifice, kidneys were obtained and processed for morphological evaluation through perfusion fixation, as previously described (39). SBP, urinary protein excretion, and serum creatinine were measured by conventional methods $(6,19)$. For the evaluation of SPS in younger animals, additional 20-week-old Wistar and MWF rats, and MWF rats treated with lisinopril between 10 and 20 weeks of age, were used ( $n=3 /$ group). At sacrifice, kidneys were obtained and processed through perfusion fixation, as described earlier (32).

TEM. For TEM analysis, kidney tissue was removed, cut in small pieces, and immersion fixed in $2.5 \%$ glutaraldehyde in $0.1 \mathrm{M}$ cacodylate buffer ( $\mathrm{pH} 7.4$ ) and postfixed in $1 \%$ osmium tetroxide. Fixed specimens were dehydrated through ascending grades of alcohol and embedded in Epon resin (Agar Scientific Ltd.) before observation at TEM (Morgagni 268D, FEI Co.). One-micrometer sections were stained with toluidine blue (Sigma-Aldrich) for identification of detached podocytes floating free in the Bowman's space and in the lumen of proximal tubules, and digital representative images from Wistar and MWF rats were obtained using light microscopy (ApoTome Axio Imager Z2, Zeiss). For toluidine blue staining, 3 rats per group were used and 10 glomeruli were analyzed per rat.

SEM. For SEM analysis, midcoronal sections of the kidneys were fixed in $2.5 \%$ glutaraldehyde in $0.1 \mathrm{M}$ cacodylate buffer ( $\mathrm{pH} 7.4$ ), postfixed in 1\% osmium tetroxide, and dehydrated in ethanol solutions. Samples were rinsed with liquid $\mathrm{CO}_{2}$ and treated with a critical point dryer before observation at SEM (1540 EsB, Zeiss).

Ultrastructure morphometrical analysis. Three glomeruli per animal were analyzed. Imaging fields from well-preserved areas of the glomerular capillary tuft were randomly taken at SEM. The size of slit pores was estimated on digitized images using ImageJ software (v. 1.5, NIH) as described previously (9). Quantification of pore size was performed in more than 600 pores in samples from all groups. The shape of pores was estimated by quantifying $R / r$, the ratio between major $(R)$ and minor $(r)$ pore radii. To evaluate the extension of SPS, glomerular section images were taken at TEM. Three rats per group were used. The fraction of tuft volume occupied by SPS was estimated using the point counting method, using an orthogonal grid digitally overlaid on the glomerular section image. For each glomerular section, the number of grid points hitting the SPS was counted, and the fraction of glomerular tuft area occupied by SPS was expressed as the percentage of grid points in the SPS relative to total points in the glomerular tuft. The thickness of the GBM layer and the height of SPS were measured on high-power electron micrographs by the harmonic mean. At least 180 measurements for SPS height and 100 measurements for GBM thickness were made for each experimental group, as described previously (16).

Immunoperoxidase experiments. Formalin-fixed, paraffin-embedded kidney sections $(3 \mu \mathrm{m})$ were deparaffinized, hydrated, and incubated for 5 minutes with Peroxidazed 1 (Biocare Medical) to quench endogenous peroxidases. Antigen retrieval was performed using a decloaking chamber (DCNxGen 220V, Biocare Medical) for 15 minutes at $110^{\circ} \mathrm{C}$ with BORG decloaker buffer (Biocare Medical) to increase the reactivity of antibody to antigen. After blocking with Rodent Block R (Biocare Medical), sections were incubated with mouse anti-WT-1 (sc-7385, clone F-6, dilution 1:30; Santa Cruz Biotechnology), followed by 
Mouse-on-Rat HRP-Polymer kit (MRT621G, Biocare Medical) and diaminobenzidine (Biocare Medical) substrate solution. Slides were finally counterstained with hematoxylin, dehydrated in graded alcohols, mounted with coverslips, and observed using light microscopy (ApoTome Axio Imager Z2, Zeiss). Three rats per group were used, and 10-12 glomeruli per kidney were analyzed. Negative controls were obtained by omitting the primary antibody on adjacent sections.

Immunofluorescence experiments. Expression of CD151 and $\alpha 3$ integrin was detected using immunofluorescence analysis. Kidney cryosections $(3 \mu \mathrm{m})$ were air dried and fixed with cold acetone. Antigen retrieval was performed in $10 \mathrm{mmol} / \mathrm{L}$ citrate buffer $(\mathrm{pH} \mathrm{6.0)}$ at a sub-boiling temperature for $20 \mathrm{~min}$ utes, followed by incubation with citrate buffer (20 minutes) at room temperature to enhance the reactivity of antibodies to antigens. After blocking with 3\% BSA, sections were incubated with rabbit antiCD151 (sc-33123, clone H-80, dilution 1:50; Santa Cruz Biotechnology) and mouse anti- $\alpha 3$ integrin (sc-7019, clone Ralph 3.2, dilution 1:300; Santa Cruz Biotechnology) followed by the species-specific Cy3- and FITC-conjugated secondary antibodies (111-165-144, Jackson ImmunoResearch Laboratories, and A11001, Thermo Fisher, Invitrogen, respectively). Nuclei were stained with DAPI. Negative controls were obtained by omitting primary antibodies on adjacent sections. Fluorescence was examined through an inverted confocal laser scanning microscope (LS510 Meta, Zeiss) and results subjected to semiquantitative analysis. CD151 and $\alpha 3$ integrin signals were graded by giving a score between 0 and 3 (0: no signal, 1: mild, 2: moderate, 3: strong diffusion). Colocalization analysis was performed using Pearson's correlation coefficient and Manders's overlap coefficient, which is based on the Pearson's coefficient, with average intensity values taken out of the mathematical expression (41). Both coefficients were calculated from red and green channels of immunofluorescence images after adjusting the threshold to minimize background noise by using an ImageJ plugin (JACoP) (42). Ten glomeruli for each animal were imaged and subjected to the colocalization analysis.

Mathematic modeling of hydraulic resistance of the glomerular capillary wall. We numerically simulated water flow through the GFB under normal and disease conditions, as well as following treatment. Briefly, to estimate the total and relative contribution of every structural layer of GFB, we followed the approach of Drumond and Deen (40), using CFD to determine velocity and pressure fields across the GFB. We first generated 3D surface models, one for each experimental group, by considering a parametric model of a 3D unit cell of GFB based on a single FS, which is periodic in the $x$ and $y$ directions, while filtration occurs in the positive $z$ direction (Figure 3). The model comprises the 3 layers that jointly determine its functional properties: ENL, GBM, and epithelial layer (EPL), as shown in Figure 3, B and C. The model was enriched with detailed dimensional data such as ENL thickness (40) and fenestrae $(11,12)$, EPL slit pore size and distribution measured in this study, as well as glomerular ultrafiltration parameters $(14,26)$, as reported in Table 4 . To estimate the contribution of the SPS to the total hydraulic resistance of the glomerular capillary wall, we used the model previously described in detail by Neal and coworkers (32). Using this model, the authors showed that resistance through the SPS depends on the diameter and length of SEPs, modulating pressure downstream of the GFB. Since direct quantification of the ultrastructural geometrical parameters of the SPS and SEP is very difficult to obtain, we performed sensitivity analysis to estimate the extension of SPS area $\left(\mathrm{A}_{\mathrm{SPS}}\right)$ covering the FSA as $\varepsilon=\mathrm{A}_{\mathrm{SPS}} / \mathrm{FSA}$, comparing calculated and measured values of total hydraulic resistance of the capillary wall, derived from previously reported micropuncture data (14), using different values of SEP diameter and length. From this sensitivity analysis, we derived the value of $\varepsilon$ for the 3 groups of rats, as well as an estimate of the hydraulic pressure inside the SPS. Detailed information about the modeling approach of the overall hydraulic resistance of the GFB, as well as assumption of input parameters, is presented in the supplemental material.

Statistics. Data were analyzed using GraphPad Prism software (v. 6.02, GraphPad Software), using 1-way ANOVA with Tukey's multiple-comparisons post hoc test to compare the differences between groups. The statistical significance level was defined as $P<0.05$. Results are expressed as mean $\pm \mathrm{SD}$ or median (IQR).

Study approval. All procedures involving animals were performed in accordance with institutional guidelines in compliance with national (D.L.n.26, March 4, 2014) and international laws and policies (European Union directive 2010/63/EU "on the protection of animals used for scientific purposes") and were approved by the Institutional Animal Care and Use Committees of Istituto di Ricerche Farmacologiche Mario Negri IRCCS. 


\section{Author contributions}

AR conceived and supervised the project, analyzed data, and wrote the manuscript; SC conceived the idea, designed and performed the experiments, analyzed data, and wrote the manuscript; BEI designed and performed the 3D modeling and CFD analysis and contributed to analyzing the data and writing the manuscript; ST supervised the project and contributed to writing the manuscript; PR performed immunofluorescence experiments; $\mathrm{AB}$ supervised the project and contributed to writing the manuscript; GR conceived and supervised the project and contributed to writing the manuscript.

\section{Acknowledgments}

The authors are indebted to Daniela Macconi and Fabio Sangalli for systemic parameters evaluations. We thank Kerstin Mierke and Rubina Novelli for English language editing. SC and PR are recipients of grants from the Fondazione Aiuti per la Ricerca sulle Malattie Rare (ARMR).

Address correspondence to: Sara Conti, Istituto di Ricerche Farmacologiche Mario Negri IRCCS, Centro Anna Maria Astori, Science and Technology Park Kilometro Rosso, Via Stezzano 87, 24126 Bergamo, Italy. Phone: 035.4213110; Email: sara.conti@marionegri.it.

1. [No authors listed]. The hidden epidemic: worldwide, over 850 million people suffer from kidney diseases. ERA-EDTA. http:// www.era-edta.org/press/180626_Prevalence_Data_Project.pdf. Accessed June 5, 2020.

2. Benigni A, Gagliardini E, Remuzzi G. Changes in glomerular perm-selectivity induced by angiotensin II imply podocyte dysfunction and slit diaphragm protein rearrangement. Semin Nephrol. 2004;24(2):131-140.

3. Kawachi H, Miyauchi N, Suzuki K, Han GD, Orikasa M, Shimizu F. Role of podocyte slit diaphragm as a filtration barrier. Nephrology (Carlton). 2006;11(4):274-281.

4. Tryggvason K, Wartiovaara J. Molecular basis of glomerular permselectivity. Curr Opin Nephrol Hypertens. 2001;10(4):543-549.

5. Remuzzi A, Puntorieri S, Mazzoleni A, Remuzzi G. Sex related differences in glomerular ultrafiltration and proteinuria in Munich-Wistar rats. Kidney Int. 1988;34(4):481-486.

6. Remuzzi A, et al. Pathophysiologic implications of proteinuria in a rat model of progressive glomerular injury. Lab Invest. 1992;67(5):572-579.

7. Fassi A, et al. Progressive glomerular injury in the MWF rat is predicted by inborn nephron deficit. J Am Soc Nephrol. 1998;9(8):1399-1406.

8. Macconi D, et al. Pathophysiologic implications of reduced podocyte number in a rat model of progressive glomerular injury. Am J Pathol. 2006;168(1):42-54.

9. Gagliardini E, Conti S, Benigni A, Remuzzi G, Remuzzi A. Imaging of the porous ultrastructure of the glomerular epithelial filtration slit. J Am Soc Nephrol. 2010;21(12):2081-2089.

10. Rodewald R, Karnovsky MJ. Porous substructure of the glomerular slit diaphragm in the rat and mouse. J Cell Biol. $1974 ; 60(2): 423-433$.

11. Rice WL, et al. High resolution helium ion scanning microscopy of the rat kidney. PLoS One. 2013;8(3):e57051.

12. Tsuji K, et al. Re-characterization of the glomerulopathy in CD2AP deficient mice by high-resolution helium ion scanning microscopy. Sci Rep. 2017;7(1):8321.

13. Deen WM, Lazzara MJ, Myers BD. Structural determinants of glomerular permeability. Am J Physiol Renal Physiol. 2001;281(4):F579-F596.

14. Remuzzi A, Puntorieri S, Battaglia C, Bertani T, Remuzzi G. Angiotensin converting enzyme inhibition ameliorates glomerular filtration of macromolecules and water and lessens glomerular injury in the rat. J Clin Invest. 1990;85(2):541-549.

15. Remuzzi A, Fassi A, Bertani T, Perico N, Remuzzi G. ACE inhibition induces regression of proteinuria and halts progression of renal damage in a genetic model of progressive nephropathy. Am J Kidney Dis. 1999;34(4):626-632.

16. Iordache BE, Imberti O, Foglieni C, Remuzzi G, Bertani T, Remuzzi A. Effects of angiotensin-converting enzyme inhibition on glomerular capillary wall ultrastructure in MWF/Ztm rats. J Am Soc Nephrol. 1994;5(6):1378-1384.

17. Yang HC, Zuo Y, Fogo AB. Models of chronic kidney disease. Drug Discov Today Dis Models. 2010;7(1-2):13-19.

18. Remuzzi G, Benigni A, Remuzzi A. Mechanisms of progression and regression of renal lesions of chronic nephropathies and diabetes. J Clin Invest. 2006;116(2):288-296.

19. Macconi D, et al. Podocyte repopulation contributes to regression of glomerular injury induced by ACE inhibition. Am J Pathol. 2009;174(3):797-807.

20. Salmon AH, et al. Evidence for restriction of fluid and solute movement across the glomerular capillary wall by the subpodocyte space. Am J Physiol Renal Physiol. 2007;293(6):F1777-F1786.

21. Neal CR, Crook H, Bell E, Harper SJ, Bates DO. Three-dimensional reconstruction of glomeruli by electron microscopy reveals a distinct restrictive urinary subpodocyte space. J Am Soc Nephrol. 2005;16(5):1223-1235.

22. Sachs N, et al. Kidney failure in mice lacking the tetraspanin CD151. J Cell Biol. 2006;175(1):33-39.

23. Chen CA, Hwang JC, Guh JY, Chang JM, Lai YH, Chen HC. Reduced podocyte expression of alpha3beta1 integrins and podocyte depletion in patients with primary focal segmental glomerulosclerosis and chronic PAN-treated rats. J Lab Clin Med. 2006;147(2):74-82.

24. Elmore S. Apoptosis: a review of programmed cell death. Toxicol Pathol. 2007;35(4):495-516.

25. Helfrich MH, Ralston SH, eds. Bone Research Protocols. Totowa, New Jersey, USA: Humana Press; 2003. Methods in Molecular 
Medicine; book 80.

26. Maddox DA, et al. Determinants of glomerular filtration in experimental glomerulonephritis in the rat. J Clin Invest. 1975;55(2):305-318

27. Blumenthal A, Giebel J, Ummanni R, Schlüter R, Endlich K, Endlich N. Morphology and migration of podocytes are affected by CD151 levels. Am J Physiol Renal Physiol. 2012;302(10):F1265-F1277.

28. Chattopadhyay N, Wang Z, Ashman LK, Brady-Kalnay SM, Kreidberg JA. alpha3beta1 integrin-CD151, a component of the cadherin-catenin complex, regulates PTPmu expression and cell-cell adhesion. J Cell Biol. 2003;163(6):1351-1362.

29. Levy S, Shoham T. Protein-protein interactions in the tetraspanin web. Physiology (Bethesda). 2005;20:218-224.

30. Baleato RM, Guthrie PL, Gubler MC, Ashman LK, Roselli S. Deletion of CD151 results in a strain-dependent glomerular disease due to severe alterations of the glomerular basement membrane. Am J Pathol. 2008;173(4):927-937.

31. Kriz W, Lemley KV. A potential role for mechanical forces in the detachment of podocytes and the progression of CKD. $J A m$ Soc Nephrol. 2015;26(2):258-269.

32. Neal CR, et al. Glomerular filtration into the subpodocyte space is highly restricted under physiological perfusion conditions. Am J Physiol Renal Physiol. 2007;293(6):F1787-F1798

33. Kretzler M. Regulation of adhesive interaction between podocytes and glomerular basement membrane. Microsc Res Tech . 2002;57(4):247-253.

34. Lennon R, Randles MJ, Humphries MJ. The importance of podocyte adhesion for a healthy glomerulus. Front Endocrinol (Lausanne). 2014;5:160

35. Pozzi A, et al. Beta1 integrin expression by podocytes is required to maintain glomerular structural integrity. Dev Biol. 2008;316(2):288-301.

36. Has C, et al. Integrin $\alpha 3$ mutations with kidney, lung, and skin disease. N Engl J Med. 2012;366(16):1508-1514.

37. Karamatic Crew V, et al. CD151, the first member of the tetraspanin (TM4) superfamily detected on erythrocytes, is essential for the correct assembly of human basement membranes in kidney and skin. Blood. 2004;104(8):2217-2223.

38. Sachs N, et al. Blood pressure influences end-stage renal disease of Cd151 knockout mice. J Clin Invest. 2012;122(1):348-358.

39. Macconi D, et al. Effect of angiotensin-converting enzyme inhibition on glomerular basement membrane permeability and distribution of zonula occludens-1 in MWF rats. J Am Soc Nephrol. 2000;11(3):477-489.

40. Drumond MC, Deen WM. Structural determinants of glomerular hydraulic permeability. Am J Physiol. 1994;266(1 pt 2):F1-12.

41. Manders EM, Stap J, Brakenhoff GJ, van Driel R, Aten JA. Dynamics of three-dimensional replication patterns during the S-phase, analysed by double labelling of DNA and confocal microscopy. J Cell Sci. 1992;103 (pt 3):857-862.

42. Bolte S, Cordelières FP. A guided tour into subcellular colocalization analysis in light microscopy. J Microsc. 2006;224(pt 3):213-232. 